\title{
Die Kommunikation zwischen Kirche und UNESCO
}

von François Russo

\section{Zur Geschichte}

Ehe man die Kommunikation zwischen Kirche und UNESCO heute beschreibt, schildert man am besten zunächst ihre Entstehung. Dadurch entsteht ein besseres Verständnis.

Man könnte sagen, die erste Kommunikation der Kirche mit der UNESCO kam spontan, auf nichtoffiziellem Weg zustande. Und zwargeschah das gleich bei Gründung der Organisation, deren erste Generalversammlung Ende 1946 stattfand. Ein für Auslandsprobleme sehr aufgeschlossener Priester, Abbé Jean Rupp, später Bischof von Monaco, Apostolischer Nuntius im Irak und heute Vertreter des Hl. Stuhls beiden Vereinten Nationen in Genf, der damals in Paris die Ausländer geistlich betreute, errichtete auf Bitten des Apostolischen Nuntius in Frankreich, Msgr. Roncalli, des späteren Papstes Johannes XXIII., Anfang 1947 ein bescheidenes Verbindungsbüro, gestützt auf eine Gruppe von Beratern und Korrespondenten. Damiter ihm bei dieser Aufgabe helfe, appellierte Abbé Rupp an einen seiner Freunde, einen jungen Pfadfinder, Jean Larnaud, den er von der Bewegung der Pfadfinder her kannte und zu deren Seelsorgern in Paris er gehörte. Msgr. Blanchet, damals Rektor des Institut Catholique von Paris, übernahm den Vorsitz dieses Organs.

1952 wurde das Unternehmen vervollständigt und erweitert, als von der UNESCO die Anwesenheit eines Beobachters des Hl. Stuhls bei ihr im Rang der die Staaten repräsentierenden Diplomaten akzeptiert wurde. Er war vornehmlich für die Anwesenheit bei den Versammlungen der Organisation und für die Kommunikation ihrer Dokumente vorgesehen, erhielt jedoch kein Stimmrecht. Übrigens zieht diese Präsenz des H1. Stuhls beiderUNESCO durch einen Beobachter, anders als für die Mitgliedstaaten, keine Beteiligung an den Kosten für die Tätigkeit der Organisation nach sich. Der erste Beobachter war Msgr. Roncalli. Ihm folgten bereits acht Beobachter. Heutiger Beobachter ist Msgr. Frana.

Obgleich nun die Beziehungen der Kirche zur UNESCO offiziell geworden waren, wurde doch das Verbindungsbüro, das die erste Stufe der Verwirklichung darstellte, nichtaufgelöst. Im Gegenteil, es sollte weiterhin zwar unabhängig, jedoch moralisch und konkret an den Beobachter gebunden, fortbestehen. Es sollte sich somit klarer abgrenzen und keineswegs als offizielles Organ, weder des Hl. Stuhls noch der UNESCO, in jeder Hinsicht im Dienst der Beziehungen der katholischen Welt mit der UNESCO stehen. Jean Larnaud, der seit der Gründung dort arbeitete, wurde der Generalsekretär.

Das Zentrum sollte durch seine Arbeit einen Informationsdienst sowohl von der UNESCO zu den katholischen Kreisen wie auch von diesen zur UNESCO sicherstellen.

Zu diesem Ziel wurde 1957 nach einer Reihe von Information sblättern eine periodische Publikation geschaffen, „Le Mois à l'Unesco”. Zunächst als Monatsschrift gedachtwurde sie als Vierteljahresschrift realisiert, zunächst nur in französischer, dann in drei weiteren Sprachen: deutsch, englisch und spanisch. Dieses Bulletin stellt ein wertvolles Kommu-

P. François Russo sj ist Berater des Internationalen Katholischen Zentrums für die UNESCO in Paris. 
nikationsinstrument zwischen den Katholiken und der UNESCO dar. Einerseits vermittelt es den Katholiken Informationen über die Aktivitäten der UNESCO, und zwar aus einem Geist und aus einer Sicht, die aufgrund ihres offiziellen Charakters und der Neutralität, zu der sie gezwungen sind, nicht die der Publikationen der UNESCO sein können. Vor allem ist dieses Bulletin imstande, jenen Aktivitäten der UNESCO Vorrang zu geben, die von ihrer Art hervornehmlich die Christen angehen. Andererseits mach tdieses Bulletin, von dem über 400 Exemplare den Funktionären der UNESCO sowohl an ihrem Sitz in Paris wie den zahlreichen Regionalämtern der Organisation zugehen, die UNESCO mit den Aktivitäten der Katholiken vertraut, soweit sie deren Programm betreffen, mit Aktivitäten also, von denen die UNESCO sonst nur schwer Kenntnis erhielte.

Kommen wir nun auf die Geschichte des Zentrums selbst zurück, so müssen wirgenauerhin sagen, daß dieses Zentrum, weil seine Dienste an erster Stelle die katholischen internationalen Organisationen betrafen, vor allem jene, die einen Beraterstatus bei der UNESCO hatten, 1952 vom HI. Stuhl einen Status bekam, der es zu einem Organ der Konferenz der katholischen internationalen Organisationen machte. DieserStatus erfordert vor allem, daß der Generalsekretär des Zentrums durch die Versammlung der Konferenz nach Billigung des Hl. Stuhls gewählt wird und daß beidiesem ein kirchlicher Berater designiert wird, der direkt vom Hl. Stuhl ernannt wird. Von 1953 bis 1960 war der kirchliche Berater Pater Quéginer, der alsdann Generalsuperior der Auslandsmissionen von Paris (MEP) wurde. Er starb 1977. Der heutige Berater ist Pater François Russo sj.

Zu einem viel jüngeren Zeitpunkt, nämlich 1973, sollte das CCIC (Abkürzung des ersten Namens des Zentrums: Centre Catholique International de Coordination auprès de l'Unesco (Internationales Katholisches Koordinationszentrum bei der UNESCO), der gegenwärtige Name des CCIC lautet genau: Centre Catholique International pour l'Unesco (Internationales Katholisches Zentrum für die UNESCO), ohne daß damit ein Wandel in seinem Wesen und seiner Funktion eintrat, eine wichtige Etappe seiner Entwicklung durchlaufen: damals wurde eine „Association Comité de soutien du Centre Catholique International pour l'Unesco" (eine unterstützende Ausschußvereinigung des Internationalen Katholischen Zentrums für die UNESCO) geschaffen. Ohne daß seine Verbindungen zur Konferenz modifiziert wurden, fand das Zentrum in dieser Vereinigung eine Hilfe, die seine Tätigkeit gestärkt und besser fundiert hat und die der Tatsache Ausdruck verleiht, daß es nicht nur ein Organ im Dienst der Katholischen Internationalen Organisationen ist, sondern daß es, viel weiter gefaßt, im Dienst der Gesamtheit der Katholiken steht, die in der Welt, aus persönlichen oder gemeinschaftlichen Gründen, für die Belange der UNESCO offen sind. Die Vereinigung zählt gegenwärtig 150 Mitglieder aus 80 Ländern. Ihre Mitglieder sind Persönlichkeiten, die auf Gebieten im Zuständigkeitsbereich der UNESCO Verantwortlichkeiten tragen: Erziehung, Kultur, Information, Sozialwissenschaft, Menschenrechte, Entwicklung, Wissenschaft und Technik ... Ihr Präsident ist seit ihrer Gründung Jean-Pierre Dubois-Dumée. Ihre drei Vize-Präsidenten sind: Dr. Franz-Josef Eilers svd (Aachen), Jan Lindemans und Michel Normand.

\section{Vielfalt der Kommunikationsarten der Kirche mit der UNESCO}

Die Geschichte, die wir soeben aufzeigten, hat schon deutlich gemacht, daß die Kommunikation zwischen der katholischen Welt und der UNESCO mehr als nur eine Form kennt. Diese Vielfalt wollen wir jetzt so vollständig wie möglich aufzeigen'. 
Man muß zwei große Kommunikationskategorien zwischen der katholischen Welt und der UNESCO unterscheiden. Die erste betrifft den H1. Stuhl, die zweite die anderen katholischen Bereiche (Einzelpersonen oder Institutionen).

\section{Die Beziehungen des HI. Stuhls zur UNESCO}

Die Beziehungen des HI.Stuhls zur UNESCO sind von dreierlei Art:

1. Da ist die bereits genannte ständige Präsenz eines Beobachters des Hl. Stuhls bei der UNESCO. So offiziell und diplomatisch diese Präsenz auch ist, sie bietet dem Beobachter zahlreiche Gelegenheiten zu persönlichen Kontakten nicht nur mit den Funktionären der UNESCO, insbesondere mit dem Generaldirektor, dem Prầsidenten des Exekutivrats, den General-Unterdirektoren, sondern auch mit den Delegierten der Staaten. Das trägt beträchtlich zu einem besseren Verständnis und zur Achtung des H1. Stuhls bei diesen Personen bei, so weit ihre Überzeugungen auch oft vom Katholizismus abweichen, da die UNESCO eine Weltorganisation ist. Und so diskret diese Beziehungen auch sind, sie haben dennoch oft einen ganz unmittelbar apostolischen Charakter.

2. Auf Einladungen der UNESCO werden Delegationen zu Welt- oder Regionalkonferenzen, ein Vertreter und auch Experten für Gesprächsrunden von besonderer oder mehr fachlicher Art designiert. Diese Art von Kommunikationen mit der UNESCO ist besonders wichtig, denn einerseits hat der HI. Stuhl die Möglichkeit, bei diesen Versammlungen Interventionen vorzunehmen, die oft ein beachtliches Echo finden aufgrund der Freiheit der Meinungsäußerung des H1. Stuhls, welche die Delegierten nicht haben. Sie erlaubt das Einbringen von Gesichtspunkten, die über die nationalen Interessen hinausreichen und Grundsatzfragen $\mathrm{zu}$ schwersten und oft delikatesten Fragen der Zivilisation von heute berühren, ob es nun um die Auffassung vom Menschen oder von der Gesellschaft aus christlicher Sicht geht. Andererseits sind diese Versammlungen die Gelegenheit zu noch weiter reichenden persönlichen Kontakten als jenen, die wir soeben aufgezeigt haben, da sich bei diesen Versammlungen die Delegationen der Mitgliedstaaten der UNESCO einfinden, aber auch Persönlichkeiten, die nicht dauernd mit der UNESCO in Beziehung stehen.

3. Persönliche Kontakte oder Kontakte über Botschaften zwischen dem Papst und dem Generaldirektor der UNESCO. So hat René Maheu, der lange Jahre Generaldirektor der UNESCO war, mehrere Male Paul VI. aufgesucht. Obwohl Maheu Agnostiker war, hob er die geistliche Stärkung und Bereicherung hervor, die er beim Papst gefunden habe. Aber bis heute hat der Papst die UNESCO nicht besucht, obgleich die UNESCO seit langem sein Kommen gewünscht hat. Generaldirektor M'Bow, der selbst vor einigen Monaten in Privataudienz von Papst Johannes Paul II. empfangen wurde. erklärte bei der letzten Versammlung des Exekutivrats: „Ich habe Papst Johannes Paul II. die Programme der UNESCO dargelegt; Sie wissen, daß Papst Paul VI. die Gewohnheit hatte, uns praktisch jedes Jahr zu empfangen, um über die Arbeit der UNESCO zu sprechen, und wir arbeiteten auf gewissen Gebieten wie denen der Alphabetisierung oder der Erhaltung historischer Monumente religiösen Charakters in bestimmten Ländern zusammen. Wir haben diese Fragen bei der Unterredung, die ich mit Papst Johannes Paul II. hatte, behandelt, und ich denke, daß wir eines Tages den Heiligen Vater bei der UNESCO zu Besuch haben werden, was eine große Auszeichnung für die Organisation wäre." 
1. Offizielle Beziehungen der 15 katholischen Internationalen Organisationen, die als Organisationen nicht von Regierungsseite bei der UNESCO beraten. Es handelt sich da bekanntlich um eine Beziehungsform, die allen Organen der Vereinten Nationen gemeinsam ist. Der Beraterstatus der nicht für Regierungen tätigen Organisationen, der so diesen Katholischen Internationalen Organisationen zugute kommt, stellt eine wertvolle offizielle Beziehung dar, denn sie bietet folgende Möglichkeiten:

\section{- Zugangsrecht zu den Dokumenten der UNESCO;}

- aufgrund des Beobachterstatus Teilnahme an der Generalkonferenz der UNESCO, die alle zwei Jahre stattfindet;

- Antworten auf Bitten um Rat der UNESCO zum Entwurf des Zweijahresprogramms der Organisation, das der Generalkonferenz unterbreitet wird;

- Interventionsmöglichkeiten (zwar nicht als Rechtsanspruch, doch werden die Bitten um Intervention stets angenommen) bei den verschiedenen Versammlungen, zu denen sie eingeladen werden.

- Das heißt genauer: wenngleich jede beratende Katholische Internationale Organisation offiziell zur Generalkonferenz eingeladen wird, so wird sie zu den anderen Versammlungen nur eingeladen, falls die UNESCO der Meinung ist, daß sie aufgrund ihres Tätigkeitsbereichs einen nutzbringenden Beitrag dazu leisten kann. Tatsächlich werden eine ziemlich große Anzahl von Katholischen Internationalen Organisationen auf diese Weise zu vielen, selbst sehr spezialisierten Versammlungen der UNESCO eingeladen. Bemerken wir noch, daß eine solche Möglichkeit den internationalen Organisationen, die keinen Beraterstatus haben, nicht geboten wird.

- Möglichkeiten, Subventionen zu erhalten oder Verträge zu schließen für Aktivitäten, die in den Programmrahmen der UNESCO fallen. Subventionen werden nicht für Regierungen tätigen Organisationen selten zugestanden, die keinen Beraterstatus besitzen.

2. Kontakte vielfältiger Formen des CCIC zur UNESCO, welche die Bitte um den Empfang von Informationen sehr unterschiedlicher Art umfassen (zum Beispiel Eintreten zugunsten dieser oder jener Person oder dieser oder jener katholischen Institution, ihnen Kontakte zu gewähren oder einer Bitte um Information zu entsprechen; ihnen dieses oder jenes Dokument zur Verfügung zu stellen; ihnen zu helfen, daß sie in den Nutzen einer Subvention oder eines Vertrags gelangen). Diese Aktivitäten bringen eine Überfülle an Korrespondenz mit den meisten Ländern der Welt mit sich, wie auch den Empfang zahlreicher Besuche, doch auch die Anwesenheit bei einer Vielzahl von Versammlungen der katholischen Organisationen. Aufgrund der Kenntnis, die das CCIC von den komplexen Vorgängen bei der UNESCO hat, erlaubt diese Tätigkeitsform des CCIC zahlreichen katholischen Personen und Institutionen außerhalb der Katholischen Internationalen Organisationen mit Beraterstatus, die einen direkten Kontakt zur UNESCO haben und sehr gut die Verfahrensweise kennen, eine Kommunikation mit der UNESCO, die sie selbst nur mit großen Schwierigkeiten unmittelbar wahrnehmen könnten. 
3. Kommunikation zwischen der UNESCO und den Katholiken durch die Publikationen des CCIC. Da steht an erster Stelle „Le Mois à l'Unesco”, wovon wir zuvor gesprochen haben. Dann gibt es zwei weitere jüngere Publikationen (in französicher und in englischer Sprache) für ein begrenzteres Publikum, weil sie mehr besondere, mehr fachliche Aspekte der Arbeit der UNESCO wiedergeben, die in „Le Mois à l'Unesco" nicht dargestellt werden können, da diese Publikation für ein breiteres Publikum bestimmt ist. Die eine, „CCIC Informations”, 1978 begründet, hebt zur Hauptsache ab auf Entwicklung, Menschenrechte, Erwachsenenbildung, Bedeutung der Wissenschaft und Technik in der Entwicklung, Probleme der Kommunikation und Information. Die andere, „CCIC Education”, 1979 begründet, betrifft den Einsatz der UNESCO auf dem Gebiet der formalen schulischen und universitären Erziehung.

4. Teilnahme von Katholiken an den „nationalen Kommissionen der UNESCO”. Diese Kommissionen haben zum Ziel, auf nationaler Ebene das Interesse für die UNESCO und eben dort ihren Einsatz zu fördern. Nach den Ländern in unterschiedlicher Weise designiert sind die Mitglieder dieser Kommissionen oft Repräsentanten katholischer Organe oder für die Zuständigkeitsbereiche der UNESCO qualifizierte Personen.

5. Mitgliedschaft von Katholiken in den „Clubs der UNESCO”. Diese Clubs, 2200 an der Zahl in 70 Ländern, deren Satzung von der UNESCO aufgestellt wurde, die aber frei gegründet werden, wo eine Gruppe von Personen es wünscht, sind eine Stätte zum Austausch, zu Studien und Informationen über das Wirken der UNESCO sowie zur Unterstützung ihres Wirkens. In ihnen besteht für die Katholiken eine besonders fruchtbare Möglichkeit der Kommunikation mit der UNESCO. Bemerken wir noch, daß es UNESCO-Clubs in einer Anzahl katholischer Schulen gibt.

6. Eine gewisse Anzahl katholischer Schulen gehört zum System der „assoziierten Schulen". Diese Schulen pflegen ein Unterrichtsprogramm in Sicht auf ein besseres internationales Verständnis.

\section{Die Gründe der Kommunikation der Kirche mit der UNESCO}

Wenn man Kommunikation betreibt, so hat man Gründe dafür, die Kommunikation entspricht einem Bedürfn is. Es geschieht, weil man bisweilen nur von einer Seite, aber auch, was hier der Fall ist, weil die Kommunikation ein Quell der Bereicherung ist, die jedem der beiden Partner ermöglicht, seine Berufung besser zu erfüllen, sie besser zu verwirklichen. Eine solche Kommunikation setzt eine gemeinsame Basis voraus, ein Ziel, auf das hin man sich einig ist, doch schließt sie eine Verschiedenheit nicht aus. Ganz im Gegenteil, die wechselseitige Bereicherung in der Kommunikation, so könnte man sagen, liegt gerade in diesem Unterschied.

Die gemeinsame Basis im Fall der Beziehungen der Kirche zur UNESCO liegt, was verschiedene Male sowohl von den Päpsten als auch von den Generaldirektoren der Organisation gesagt worden ist, in der gleichen Sorge um den Menschen, als einzelnen wie als Gemeinschaft, um seine Entwicklung, um seine Förderung, aus einer Überzeugung, die heute von allen aufrichtigen Menschen guten Willens geteilt wird, und zwar in Sicht auf den Frieden, die Gerechtigkeit und die Freiheit. Konkret kommt diese Konvergenz in einem gemeinsamen Interesse der Kirche und der UNESCO zum Ausdruck, besonders für die Ausweitung und den Fortschritt der Erziehung, die nicht nur Unterweisung und Ler- 
nen, sondern die Bildung des ganzen Menschen umfaßt und in dem besteht, was über den Zugang einergrößeren Anzahl von Menschen zum Lesen und Schreiben noch mehr in die Tiefe geht, durch die Unterstützung all dessen, was die Kultur fördert, wobei es um die Bewahrung und um ein volles Bewußtwerden des Wertes von Kultur-,,Monumenten" geht (Architektur, Kunstwerke, geschriebene und mündlich überlieferte Literatur, Musik...). Von Monumenten, die nicht nur in der christlichen Zivilisation, sondern auch in den anderen Zivilisationen oftmals religiösen Charakter haben. Durch gleiche Sorge um die Förderung der Wissenschaft, einerseits als Werk der Wahrheit, einer Wahrheit, die für den Christen der Widerschein der vollen Wahrheit ist, andererseits als wesentliches Instrument der Entwicklung und des Fortschritts der Völker, einer Wissenschaft, die dann in der Technik weiter wirkt. Durch Wirken in Sicht darauf hin, allen Zugang zu der Information zu geben, die sie nötig haben, um sich zu entwickeln, um mit den anderen Menschen und den anderen Völkern Kommunikation und Gemeinschaft zu pflegen. Durch Kampf gegen alles, was sich gegen die grundsätzliche Gleichheit der Menschen richtet, vor allem durch ein ständiges Bemühen in Sicht darauf hin, die Diskriminierungen und Unterdrückungen auszuschalten, die sich unter so unterschiedlichen Formen in unserer Zivilisation zeigen: politisch, wirtschaftlich, kulturell, rassistisch und auch Diskriminierung, die so häufig die Frau noch auf einem niedrigeren Lebensniveau festhält. Jedes dieser gemeinsamen Ziele kann man auf vielfältige Weise zu realisieren anstreben, wobei die Kirche und die UNESCO unter sehr abgewandelten Formen und verschieden intensiv zusammengearbeitet haben: bei Studienseminaren, Meinungskampagnen, Bildungsforen, Kolloquien über grundlegende, philosophische, soziale, pädagogische, wirtschaftliche Themen.

Diese Kommunikation der Kirche mit derUNESCO geschieht indessen in Verschiedenheit. Der Unterschied liegt wesentlich darin, daß die erste und grundlegende Sicht der Kirche religiöser Ordnung ist und daß die tiefsten Gründe für ihren Einsatz, auch wenn es sich um einen profanen handeln sollte, in ihrem Glauben an Jesus Christus, den Erlöser und Retter der Welt, liegen, während die Sicht der UNESCO das nicht tut, da sie gar nicht religiös sein kann. Gewiß verkündet und praktiziert die UNESCO die Achtung der Religionsfreiheit, und mehr als einmal hat sie Versammlungen einberufen, beidenen die Beiträge der Religionen zu den von ihr verfolgten Zielen dargelegt worden sind. Aberweil sie eine staatliche Vereinigung ist und weil in der Welt die Überzeugungen sehr verschieden, manche sogar atheistisch sind, ist die UNESCO zur Neutralität gezwungen. Diese Situation hat sicherlich zur Konsequenz, daß, wenn auf die eine oder andere Weise der katholische Glaube und das katholische Denken beider UNESCO Ausdruck finden, das doch in einer diskreten Weise geschehen muß, die der Verschiedenheit de r dort repräsentierten Meinungen mit Respekt Rechnung trägt. Man muß sehr wohl wissen, daß diese Diskretion, das Schweigen, das sie in sich schließt, die Zurückhaltung, zu der sie verpflichtet, b isweilen die Katholiken belastet, die vor der UNESCO sprechen; sie leiden darunter, daB sie die Evangelienbotschaft nicht in ihrerganzen Kraft und ihrer ganzen Klarheit verkünden können. Dennoch hat diese Lage der Dinge sie nichtgehindert, verschiedentlich ihre Überzeugungen sehr deutlich auszusprechen, ja sogar diese oderjene Praktiken, die mit dem christlichen Ideal unvereinbar sind, diese oder jene schweren Mängel am Einsatz der in den Staaten Verantwortlichen kräftig und nachdrücklich zu verurteilen.

Diese Darlegung über die Beziehungen der Kirche zur UNESCO wäre nicht vollständig, würde man nicht die verschiedenen Schwierigkeiten, auf die sie stoßen, erwähnen. Die UNESCO ist keine vollkommene Organisation. Sie hat eine Entwicklung genommen, die aus ihr einen sehr schwerfälligen Organismus macht. Ihr Einsatz ist nicht immer so 
wirksam, wie er es sein sollte; andererseits wird ihr Wirken, da sie aus einer Vereinigung von Staaten besteht, bisweilen durch politische, ihrer Arbeit entgegenstehende Erwägungen erschwert, da es normalerweise einzig im Bereich des „Geistes” liegen und den Dienst am Menschen im Auge haben sollte ohne eine andere Sorge als dessen Wohl. Von Seiten der Katholiken muß man ein noch zu wenig ausgesprochenes Interesse für die UNESCO und für das Verständnis ihres Wirkens vermerken. Die Katholiken, die unter verschiedenen Rücksichten mit derUNESCO zusammenarbeiten, haben sehr oft Mühe, sich entweder über die Leitungen der Institutionen, von denen sie abhängen, oder über die öffentliche Meinung verständlich zu machen. Fügen wir noch hinzu, daß die Not der Menschen und die Armut der Mittel es nicht zulassen, die Beziehungen der Kirche zur UNESCO so zu entwickeln, wie esnötig wäre. Dennoch erweist sich das, was gegenwärtig verwirklicht wird, als fruchtbringend und scheint eine der annehmbarsten Arten jenes tieferen Engagements der Kirche in der Welt zu sein, die so lebhaft vom II. Vatikanischen Konzil gewünscht worden ist ${ }^{2}$.

\section{Anmerkungen}

1 Unsere Darlegung beschränkt sich auf die Beziehungen der Kirche zur Unesco, doch muß vermerkt werden, daß es ähnliche Beziehungen der Kirche zu den Vereinten Nationen in New York und zu den anderen großen spezialisierten Zweigstellen der Vereinten Nationen gibt.

2 Es erscheint uns nicht angebracht, hier eine Bibliographie der zahlreichen Publikationen zu verzeichnen, die entweder einer Gesamtdarstellung der Unesco und ihres Einsatzes oder den Beziehungen der Kirche zur Unesco gewidmet sind. Zwei wichtige Publikationen seien jedoch erwähnt:

Pie XII: L'Education, la Science et la Culture. Paris 1956, 240 Seiten. Obgleich schon alt, behält dieses Werk seinen vollen Wert; Es legt in der Tat in geordneter Folge im wesentlichen das Denken des damaligen Papstes Pius XII. vor über die Gebiete, denen sich die Arbeiten und Aktivitäten der Unesco zuwenden. Das Vorwort schrieb Msgr. Montini, damals Erzbischof von Mailand, der spätere Papst Paul VI.

Maurice Barbier, op: L'Eglise catholique et l'Unesco, vingt-cinq ans de relations. Présentation de Vincent Cosmao, Paris, Centre L.J.Lebret "Foi et Développement" (9, rue Guénégaud 75006 Paris). Vervielfältigt, 151 Seiten. Eine sehr emsthafte und gut dokumentierte Studie; einige etwas ,leichtfertige” Urteile.

\section{SUMMARY}

Communications between the Church and UNESCO first developed from a Paris Coordinating Office which began in 1947 with Mgr. Roncalli, later Pope John XXIII. When, in 1952, a Permanent Observer of the Holy See with diplomatic status was appointed (the first observer also being Mgr. Roncalli), the Coordinating Office was retained as an Information Centre with an ecclesiastical advisor in the service of the International Catholic Organisations. Communication between the Church and UNESCO takes different routes. - There is the presence of the observer from the Holy See; there are delegations and experts; personal contacts and contacts through Embassies, as far as the Vatican is concerned. In addition to the oflicial relations of International Catholic Organisations with consultative status there are also contacts with the Catholic Coordinating Office through their publications, through participation of Catholics in National UNESCO Commissions, membership in UNESCO Clubs, etc. 
There ist also a considerable range of joint interest between the Church and this World Organisation, especially where these interests and concerns differ and where, on account of the spiritual dimension of the Church, some tension may be experienced. It is regretted that there is a lack of interest and appreciation for the work of UNESCO by Catholics, who, according to Vatican II, should be more committed to activities in the secular world.

\section{RÉSUMÉ}

L'auteur résume d'abord de façon historique comment se développa la communication entre l'Eglise et l'Unesco à partir du bureau de liaison de Paris, en 1947 par Msgr. Roncalli (plus tard Pape Jean XXIII), en 1952 en l'absence d'un observateur du Saint-Siège, d'ordre diplomatique (premier observateur également Msgr. Roncalli), en quoi on continua d'utiliser le bureau de liaison comme centre d'information, et certes avec un conseiller ecclésiastique (aujourd'hui P. François Russo sj) au service des organisations internationales catholiques. La communication de l'Eglise avec l'UNESCO a de nombreux aspects, cela se passe en présence de l'observateur du Saint-Siège, par l'intermédiaire de délégations et d'experts, par les contacts personnels et par l'intermédiaire des ambassades dans les cas où elles concernent le Vatican; à cela viennent s'ajouter les rapports officiels des conseillers des organisations catholiques internationales, les contacts des bureaux de liaison, la communication par leurs publications, la participation des catholiques aux commissions nationales de l'UNESCO, l'appartenance des catholiques à des clubs de l'UNESCO, etc. . . Enfin, l'auteur explique la communication entre l'Eglise et l'UNESCO par la convergence d'intérêts qui, malgré les différences face à l'Eglise, sont grands par leur dimension intellectuelle et spirituelle et pas toujours sans tension non plus. D'autre part, il regrette encore le manque d'intérêt et de compréhension pour l'action de l'UNESCO aux côtés des catholiques ce qui devrait amener l'Eglise à un plus grand engagement dans le monde, dans l'esprit de Vatican II.

\section{RESUMEN}

El autor resume primeramente como se desarrolló la comunicación entre la Iglesia y la UNESCO, desde la creación de la Oficina de Enlace en Paris, que funcionó desde 1947 encabezada por Monseñor Roncalli (más tarde Juan XXIII), hasta la presencia desde 1952 de un observador de la Santa Sede con rango diplomático (primer observador el mismo Monseñor Roncalli). La Oficina de Enlace sigue funcionando como centro de información al servicio de organizaciones católicas internationales, dirigido por un consejero eclesiástico (en la actualidad el P. François Russo, SJ). La comunicación de la Iglesia con la UNESCO es múltiple: a través de la presencia del observador de la Santa Sede, de delegaciones y expertos, y a través de contactos personales con los embajadores. A ello hay que añadir las relaciones oficiales de los consejeros de las organizaciones internacionales católicas, los contactos de la Oficina de Enlace, la comunicación a través de publicaciones, la participación de católicos en comisiones nacionales de la UNESCO, la afiliación de católicos a los clubs de la UNESCO, etc. Por último, el autor justifica la comunicación entre la Iglesia y la UNESCO en la gran convergencia de intereses, no libre de conflictos, existente a pesar de las diferencias de sus respectivas dimensiones espirituales. Por otra parte, lamenta el escaso interés y comprensión de los católicos hacia la eficacia de la UNESCO, a pesar de que, según el Vaticano II, la Iglesia debería comprometerse más en el mundo. 